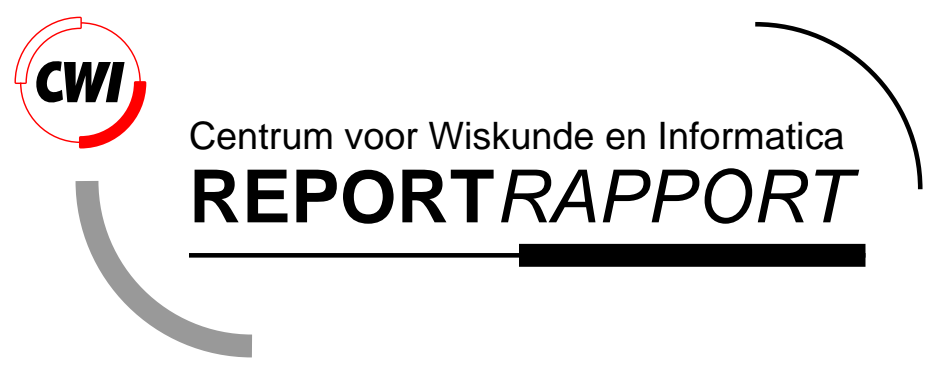

Complementarity modeling of hybrid systems

A.J. van der Schaft and J.M. Schumacher

Department of Operations Reasearch, Statistics, and System Theory

BS-R9611 1996 
Report BS-R9611

ISSN 0924-0659

CWI

P.O. Box 94079

1090 GB Amsterdam

The Netherlands

$\mathrm{CWI}$ is the National Research Institute for Mathematics and Computer Science. CWI is part of the Stichting Mathematisch Centrum (SMC), the Dutch foundation for promotion of mathematics and computer science and their applications.

SMC is sponsored by the Netherlands Organization for Scientific Research (NWO). CWI is a member of ERCIM, the European Research Consortium for Informatics and Mathematics.

Copyright @ Stichting Mathematisch Centrum P.O. Box 94079, 1090 GB Amsterdam (NL) Kruislaan 413, 1098 SJ Amsterdam (NL) Telephone +3120 5929333 Telefax +3120 5924199 


\title{
Complementarity Modeling of Hybrid Systems
}

\author{
A.J. van der Schaft \\ Systems and Control Group, Department of Mathematics, University of Twente \\ P.O. Box 217, 7500 AE Enschede, The Netherlands, and \\ CWI, P.O. Box 94079, 1090 GB Amsterdam, The Netherlands \\ a.j.vanderschaft@math. utwente.nl \\ J.M. Schumacher \\ CWI \\ P.O. Box 94079, 1090 GB Amsterdam, The Netherlands, and \\ Tilburg University, CentER and Department of Economics, \\ P.O. Box 90153, 5000 LE Tilburg, The Netherlands \\ Hans.Schumacher@cwi.nl
}

\begin{abstract}
A complementarity framework is described for the modeling of certain classes of mixed continuous/discrete dynamical systems. The use of such a framework is well-known for mechanical systems with inequality constraints, but we give a more general formulation which applies for instance also to systems with relays in a feedback loop. The main theoretical results in the paper are concerned with uniqueness of smooth continuations; the solution of this problem requires the construction of a map from the continuous state to the discrete state. A crucial technical tool is the so-called linear complementarity problem (LCP); we introduce various generalizations of this problem. Specific results are obtained for Hamiltonian systems, passive systems, and linear systems.
\end{abstract}

AMS Subject Classification (1991): 34A12, 34A09, 58F05, 68U20, 93B12.

Keywords \& Phrases: hybrid systems, differential/algebraic equations, inequality constraints, complementarity problem.

Note: This report has been submitted for publication elsewhere.

\section{INTRODUCTION}

The mixing of continuous and discrete dynamics on the one hand and of plant and controller dynamics on the other hand can lead to many different settings for hybrid control systems. In some cases the continuous dynamics may reside completely in the plant and the discrete dynamics may be associated completely with the controller, but in other cases the controller may be of a mixed discrete/continuous nature, in still other cases the plant itself may be hybrid, and one may also have situations where both plant and controller are of mixed type. The interaction between plant and controller may have a discrete or a continuous nature; sometimes the distinction may be not so clear, as in the classical case of bang-bang control in which the scalar control input is a priori allowed to be piecewise continous, but effectively assumes only two values. The controlled system may actually be subject to various inputs (controls and disturbances) which may be of different nature, and also the measurements and the purposes of control may be of discrete or of continuous nature or both.

In this paper we shall be mainly concerned with the description of the discontinuous dynamics of systems that switch between various modes as a result of state events; such events may be triggered by a control mechanism or by the nature of the plant (the distinction may not always be clear and is 


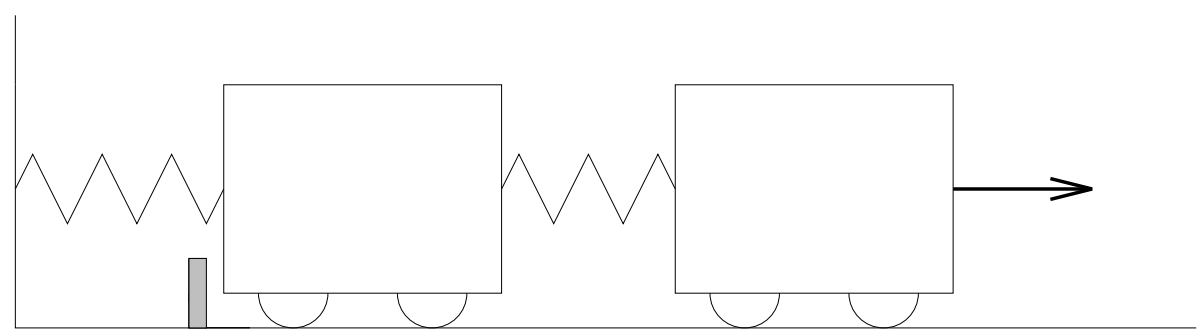

Figure 1. A system with state events.

immaterial in a closed-loop situation). To motivate the development, let us consider the following very simple example of such a hybrid system with a continuous input (see Fig. 1). Two carts are connected to each other and to a fixed wall by springs. The motion of the left cart is restricted by a purely non-elastic stop. A control force can be exerted on the right cart. For simplicity, we shall normalize all constants to 1 and let the springs be linear, and we shall assume that the stop is placed at the equilibrium position of the left cart. An 'event' takes place when the left cart hits the stop or when it is pulled away from a position at the stop; such events mark a change in nature of the dynamical system as seen by the input. When an event takes place, the system switches from 'constrained mode' to 'unconstrained mode' or vice versa; these two modes correspond to the two discrete states of the system. Note that the function $v(t)$ which we have called an input may be viewed as a control or as a disturbance; in a situation in which the system depicted in Fig. 1 is part of a larger system, we may also view $v(t)$ as just a connecting variable.

We shall now discuss the dynamics of the system in the example. It is not difficult to write equations of motion for each of the two modes separately. Let $x_{1}(t)$ and $x_{2}(t)$ represent the deviations of the left and the right cart respectively from their equilibrium positions, and let $x_{3}(t)$ and $x_{4}(t)$ denote the corresponding velocities. In the unconstrained mode the equations are the ones that would hold if there were no block:

$$
\begin{aligned}
& \dot{x}_{1}(t)=x_{3}(t) \\
& \dot{x}_{2}(t)=x_{4}(t) \\
& \dot{x}_{3}(t)=-2 x_{1}(t)+x_{2}(t) \\
& \dot{x}_{4}(t)=x_{1}(t)-x_{2}(t)+v(t) .
\end{aligned}
$$

The equations of motion in the constrained mode are the ones that would hold if the first cart were nailed to the block:

$$
\begin{aligned}
& x_{1}(t)=0 \\
& \dot{x}_{2}(t)=x_{4}(t) \\
& x_{3}(t)=0 \\
& \dot{x}_{4}(t)=-x_{2}(t)+v(t) .
\end{aligned}
$$

To give a complete description of the hybrid system, one also needs to specify under what conditions events take place and what the effects of such events will be. Under the assumption of inelastic collision, one can argue that a transition from the unconstrained mode to the constrained mode will occur at times $t_{0}$ when the following Boolean expression in terms of equality and inequality conditions on the state variables evaluates to TRUE:

$$
\left(x_{1}\left(t_{0}\right)=0\right) \wedge\left(\left(x_{3}\left(t_{0}\right)<0\right) \vee\left(\left(\left(x_{3}\left(t_{0}\right)=0\right) \vee\left(\left(x_{2}\left(t_{0}\right)<0\right) \vee\left(\left(x_{2}\left(t_{0}\right)=0\right) \wedge\left(x_{4}\left(t_{0}\right)<0\right)\right)\right)\right)\right)\right)
$$


Moreover, when this event takes place the variable $x_{3}$ is reset to zero, whereas the other continuous state variables keep the values that they had just before the event. A transition from the constrained mode to the unconstrained mode will take place at times $t_{0}$ when the following expression evaluates to TRUE:

$$
\left(x_{2}\left(t_{0}\right)=0\right) \wedge\left(x_{4}\left(t_{0}\right)>0\right) .
$$

This event produces no jumps in the continuous state variables. Note that it is possible that the conditions for a transition from the constrained mode to the unconstrained mode are satisfied immediately after a transition from the unconstrained mode to the constrained mode has taken place; in such cases there are two events in one time instant (in a well-defined order).

The equations and conditions given above are already fairly complicated although they come from a very simple example. In many cases the formulation of equations of motion in the above explicit form, including all the condition/event rules, will be a formidable task, and there is a clear need for devices that enable the modeler to work in what might be called a 'high-level language'. This language should be such that it allows automatic translation to a 'low-level language' which provides the explicit forms as in the above, and which can be used as a basis for simulation and other purposes. The situation is not unlike that of analytical mechanics, where one avoids having to write down equations of motion for each mechanical system from scratch by introducing 'principles' and 'formalisms' that can be written down easily, and from which the explicit equations of motion can be written down in a perhaps tedious but in principle straightforward way. It is the purpose of this paper to discuss such a formalism that may be used for certain classes of hybrid systems. Given the vastness of the area covered by the term 'hybrid dynamical systems', there is no reason to assume that there will be any modeling principle having uniform validity for all hybrid systems; on the contrary, one should expect that powerful principles can only be formulated for fairly limited classes. In view of the success of studies of classes of dynamical systems with very special properties (linear systems, Hamiltonian systems, planar systems, singularly perturbed systems, etc.), one shouldn't be too unhappy with such limitations.

We call the formalism that will be discussed in this paper the complementarity formalism because it is based on complementarity ideas (in the sense to be explained below). Roughly speaking, the modeling principle that is used here applies to hybrid systems that arise from the combination of inequality constraints with variational principles; examples include mechanical systems, optimization problems, and electrical networks. A number of specific applications will be discussed below, which show that the extent of complementarity modeling is larger than one might think at first. In this paper we shall be mainly concerned with the issue of well-posedness, in the sense of existence and uniqueness of solutions for each given input $v(\cdot)$. This is clearly a basic question to deal with in any dynamical system. One of the benefits that one may expect from the formulation of modeling principles is that they will make it possible to verify well-posedness for at least some classes of hybrid systems. In this paper we shall concentrate on the uniqueness of smooth continuations, generalizing (in this respect) the earlier results in [16] which were limited to the case of systems with only two discrete states. The generalization is made possible by the use of what we call the 'dynamic complementarity problem', which is an extension of the linear complementarity problem (LCP) that has been studied widely in the mathematical programming community (see for instance [3]). It should be noted that proving well-posedness is not a merely academic exercise; besides providing a test of integrity of the formalism, any constructive method used in the proof can be put to use in algorithms for the computation of solutions.

The paper is structured as follows. We state the complementarity formalism in the next section and show how it can be applied to some situations that might appear at first sight not to be covered by the formalism. Specifically, we shall discuss Coulomb friction, relays, and input saturation. In section 3, we introduce the dynamic complementarity problem (DCP) and derive a general result on uniqueness of smooth continuations. We then apply this result to a number of particular cases in section 4. Conclusions follow in section 5 . 


\section{COMPLEMENTARITY MODELING}

We consider systems which are described by general differential-algebraic equations

$$
F(z(t), \dot{z}(t))=0, \quad z \in \mathbb{R}^{N}
$$

together with a complementary set of inequality constraints defined as follows. Define two mappings

$$
\begin{array}{ll}
y(t)=H_{1}(z(t)), & y \in \mathbb{R}^{k} \\
u(t)=H_{2}(z(t)), & u \in \mathbb{R}^{k}
\end{array}
$$

(note, in first instance $u$ and $y$ should not be interpreted as inputs and outputs, see however below) and consider the "complementary-slackness conditions" (the terminology stems from optimization theory)

$$
y(t) \geq 0, \quad u(t) \geq 0, \quad y^{T}(t) u(t)=0
$$

where the inequalities are understood componentwise. The conditions on $y(t)$ and $u(t)$ imply that for each index $i$ in the index set $K:=\{1, \cdots, k\}$ and each time $t$ we must have either $y_{i}(t)=0$ and $u_{i}(t) \geq 0$, or $u_{i}(t)=0$ and $y_{i}(t) \geq 0$. Thus for every subset $I \subset K$ we obtain a different set of DAE's

$$
\begin{aligned}
& F(z(t), \dot{z}(t))=0 \\
& H_{1 i}(z(t) \\
& H_{2 i}(z(t)) \quad 0, \quad i \in I \\
& =0, \quad i \in K \backslash I
\end{aligned}
$$

together with feasibility conditions

$$
\begin{aligned}
& H_{1 i}(z(t)) \geq 0, \quad i \in K \backslash I \\
& H_{2 i}(z(t)) \geq 0, \quad i \in I .
\end{aligned}
$$

The dynamics described by (2.4) will be called a mode of the hybrid system; the mode corresponding to the subset $I$ will be simply denoted as "mode $I$ ". It follows that we have obtained a hybrid (or multi-mode) system with, in principle, $2^{k}$ different modes, which each have to satisfy a set of additional feasibility conditions (2.5). These hybrid systems have been called "complementary-slackness systems" in [16].

A point $z_{0} \in \mathbb{R}^{N}$ is said to be consistent for mode $I$ if there exists a continuously differentiable solution of (2.4) passing through $z_{0}$; the point $z_{0}$ is said to be feasible for mode $I$ if in addition the inequality constraints (2.5) are satisfied for $z(t)=z_{0}$. The set of all points that are consistent for mode $I$ will be denoted by $\mathcal{V}_{I}$. Every mode $I$ may describe a dynamics with continuous inputs; i.e. we may be able to represent $z$ (satisfying (2.4)) through $\left(x_{I}, v_{I}\right)$, with $x_{I}$ the state of mode $I$ and $v_{I}$ the inputs of mode $I$. In the sequel, however, we will mainly concentrate on the case that every mode $I$ is autonomous, i.e. for every consistent point $z_{0}$ there exists only one solution $z(t)$ of (2.4) (meaning that the vector $v_{I}$ is void). A special class of complementary-slackness systems which is especially amenable for analysis (in the sense that standard concepts and tools from systems and control theory can be exploited), is obtained by replacing $(2.1-2.2)$ by the equations of an input-state-output system

$$
\begin{aligned}
& \dot{x}(t)=f(x(t), u(t)) \\
& y(t)=h(x(t), u(t))
\end{aligned}
$$

and adding to (2.6) the complementary-slackness conditions

$$
y(t) \geq 0, \quad u(t) \geq 0, \quad y^{T}(t) u(t)=0 .
$$


We will call (2.6-2.7) a "semi-explicit complementary-slackness system". There are many examples of (semi-explicit) complementary-slackness systems. Perhaps the most immediate examples are electrical networks with diodes and mechanical systems subject to geometric inequality constraints, as already demonstrated in [16]. Indeed, the equations of a (linear) electrical network with diodes can be obtained by first replacing the diodes by external ports, leading to a port-controlled representation

$$
\begin{aligned}
\dot{x}(t) & =A x(t)+B u(t) \\
y(t) & =C x(t)+D u(t)
\end{aligned}
$$

with $u_{i}$ denoting either the current or voltage at the $i$-th port, and $y_{i}$ denoting accordingly either the voltage or the current. Connecting the diodes to the external ports will produce equations $u_{i}=$ $-V_{i}, y_{i}=I_{i}$, or $u_{i}=I_{i}, y_{i}=-V_{i}$. By finally adding the ideal diode characteristics

$$
V_{i} \leq 0, \quad I_{i} \geq 0, \quad V_{i} I_{i}=0
$$

one then obtains a (linear) input-output complementary-slackness system (2.6-2.7). Mechanical systems with geometric inequality contraints (such as the simple example of Figure 1) are given by equations of the following form (see [16]), in which $\frac{\partial H}{\partial p}$ and $\frac{\partial H}{\partial q}$ denote column vectors of partial derivatives, and the time arguments of $q, p, y$, and $u$ have been omitted for brevity:

$$
\begin{array}{rlrl}
\dot{q} & =\frac{\partial H}{\partial p}(q, p) & & q \in \mathbb{R}^{n}, p \in \mathbb{R}^{n} \\
\dot{p} & =-\frac{\partial H}{\partial q}(q, p)+\frac{\partial C^{T}}{\partial q}(q) u, & & u \in \mathbb{R}^{k} \\
y & =C(q), & & y \in \mathbb{R}^{k} \\
y \geq 0, \quad u \geq 0, \quad y^{T} u=0 . & &
\end{array}
$$

Here, $C(q) \geq 0$ is the column vector of geometric inequality constraints, and $u \geq 0$ is the vector of Lagrange multipliers producing the constraint force vector $\frac{\partial C^{T}}{\partial q}(q) u$. $\left(\frac{\partial C^{T}}{\partial q}\right.$ denotes an $n \times k$ matrix with $i$-th column given by $\frac{\partial C_{i}}{\partial q}$.) The complementary-slackness conditions in this case express that the $i$-th component of $u_{i}$ can be only non-zero if the $i$-th constraint is active, that is, $y_{i}=C_{i}(q)=0$. Furthermore, $u_{i} \geq 0$ since the constraint forces will be always pushing in the direction of rendering $y_{i}$ non-negative. This basic principle of handling geometric inequality constraints can be found e.g. in $[13,9]$, and dates back to Fourier and Farkas. $H(q, p)$ (the Hamiltonian) denotes the total energy, generally given as the sum of a kinetic energy $\frac{1}{2} p^{T} M^{-1}(q) p$ (where $M(q)$ denotes the mass matrix, depending on the configuration vector $q$ ) and a potential energy $V(q)$. The semi-explicit complementary-slackness system (2.10) is called a Hamiltonian complementary-slackness system, since the dynamics of every mode is Hamiltonian [16]. In particular, every mode is energy-conserving (since the constraint forces are workless); it should be noted though that the model could be easily extended to mechanical systems with dissipation by replacing the second set of equations of (2.10) by

$$
\dot{p}=-\frac{\partial H}{\partial q}(q, p)-\frac{\partial R}{\partial \dot{q}}(\dot{q})+\frac{\partial C^{T}}{\partial q}(q) u
$$

where $R(\dot{q})$ denotes a Rayleigh dissipation function.

We will now discuss some less "obvious" examples of complementary-slackness systems. Our first example concerns the modeling of Coulomb friction for mechanical systems. Coulomb friction is described by the ideal characteristic in the $(v, F)$-plane depicted in Fig. 2. Here $v$ denotes the velocity, and $F$ the friction force which is equal to a constant $F_{c}$ for $v>0$, or $-F_{c}$ for $v<0$, while $-F_{c} \leq F \leq F_{c}$ for $v=0$. Clearly, a single Coulomb friction element has three modes, and it is not immediate how 


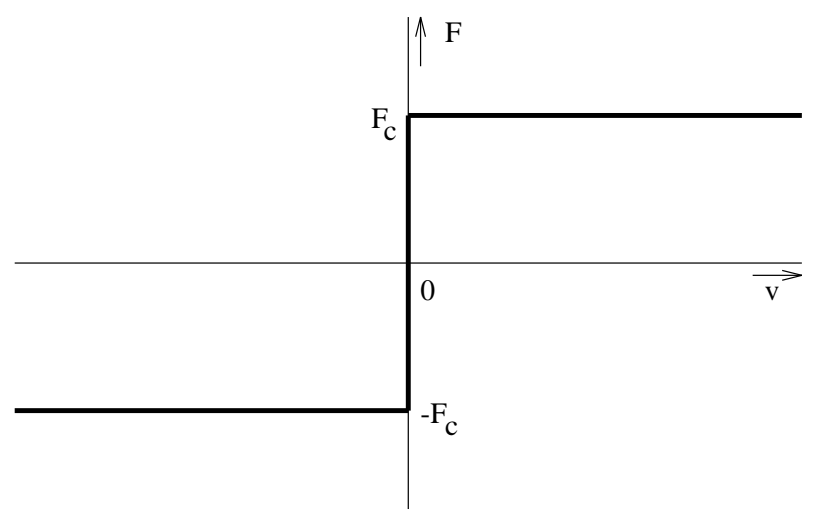

FIGURE 2. Coulomb friction characteristic.

a mechanical system with Coulomb friction elements can be modeled as a complementary-slackness system. Introduce however two auxiliary variables $\xi_{1}, \xi_{2}$, and define $y=\left(y_{1}, y_{2}\right)^{T}, u=\left(u_{1}, u_{2}\right)^{T}$ as

$$
\begin{array}{ll}
y_{1}:=\xi_{1}, & u_{1}:=F_{c}-F \\
y_{2}:=\xi_{2}, & u_{2}:=F_{c}+F .
\end{array}
$$

Furthermore, impose the complementary-slackness conditions

$$
y \geq 0, \quad u \geq 0, \quad u^{T} y=0,
$$

and the algebraic constraint

$$
v=\xi_{1}-\xi_{2},
$$

where $v$ is a generalized velocity corresponding to a mechanical system

$$
\begin{aligned}
\dot{q} & =\frac{\partial H}{\partial p}(q, p), \quad q, p \in \mathbb{R}^{n} \\
\dot{p} & =-\frac{\partial H}{\partial q}(q, p)-\frac{\partial R}{\partial \dot{q}}(\dot{q})-A(q) F \\
v & =A^{T}(q) \dot{q}
\end{aligned}
$$

for some $n$-dimensional vector $A(q)$. Equations (2.15) express that $v$ is the generalized velocity "causing" the Coulomb friction force $F$. The equations $(2.12-2.15)$ define a complementary-slackness system of the general form (2.1-2.3) (with $\left.z=\left(q, p, \xi_{1}, \xi_{2}, v, F\right)\right)$ having four modes described by the tableau

\begin{tabular}{c|c|c} 
& $y_{1}=0$ & $y_{1} \geq 0$ \\
& $u_{1} \geq 0$ & $u_{1}=0$ \\
\hline$y_{2}=0$ & $v=0$ & $v \geq 0$ \\
$u_{2} \geq 0$ & $-F_{c} \leq F \leq F_{c}$ & $F=F_{c}$ \\
\hline$y_{2} \geq 0$ & $v \leq 0$ & $F_{c}=-F_{c}$ \\
$u_{2}=0$ & $F=-F_{c}$ & not feasible
\end{tabular}

which indeed yields the three modes of Figure 2. It is clear that mechanical systems with multiple Coulomb friction elements can be modeled in the same way as complementary-slackness systems by 


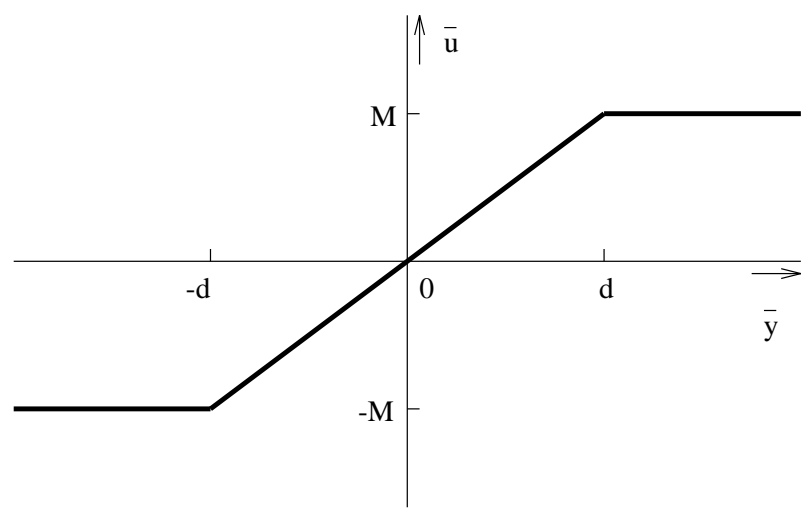

FIGURE 3. Saturation characteristic.

repeating the above procedure for every Coulomb friction element, leading to $2 \ell$-dimensional $y$ - and $u$-vectors in case of $\ell$ Coulomb friction elements.

The same modeling as complementary-slackness systems can be applied to control systems (linear or nonlinear) containing ideal relays. Relays without deadzone have the same characteristics as in Figure 2 , with $v$ replaced by a measured output $\bar{y}$, and $F$ replaced by a control input $\bar{u}$. Ideal saturation elements can be modeled quite similarly. Again we introduce two auxiliary variables $\xi_{1}, \xi_{2}$; define

$$
\begin{array}{ll}
y_{1}:=\xi_{1}, & u_{1}:=M-\bar{u} \\
y_{2}:=\xi_{2}, & u_{2}:=M+\bar{u}
\end{array}
$$

together with an algebraic constraint (compare with (2.14))

$$
\bar{y}=\xi_{1}-\xi_{2}+\frac{d}{M} \bar{u}
$$

and impose the complementary-slackness conditions $y \geq 0, u \geq 0, u^{T} y=0$. As in the tableau for Coulomb friction this yields three feasible modes, corresponding to the three linear parts of the characteristic given in Figure 3. Of course, this raises the interesting question which class of multimode systems actually can be formalized as complementary-slackness systems; it seems that most piecewise linear characteristics can be modeled this way. Indeed, as we will see in the next section, there is a close connection between complementary-slackness systems and the linear complementarity problem (LCP) of mathematical programming. Since the LCP is known to be related to piecewise linear functions (see for instance [4]), it is likely that a close connection exists between systems with piecewise linear characteristics and complementary-slackness systems.

As a final illustration of the range of complementarity modelling we briefly give the complementarityslackness modelling of a relay with deadzone as in Figure 4. We introduce five auxiliary variables $\xi_{1}, \xi_{2}, \xi_{3}, \xi_{4}, \eta$, and define

$$
\begin{array}{ll}
y_{1}:=\xi_{1} & u_{1}:=M-\bar{u} \\
y_{2}:=\xi_{2} & u_{2}:=M+\bar{u} \\
y_{3}:=d-\eta & u_{3}:=\xi_{3} \\
y_{4}:=d+\eta & u_{4}:=\xi_{4}
\end{array}
$$




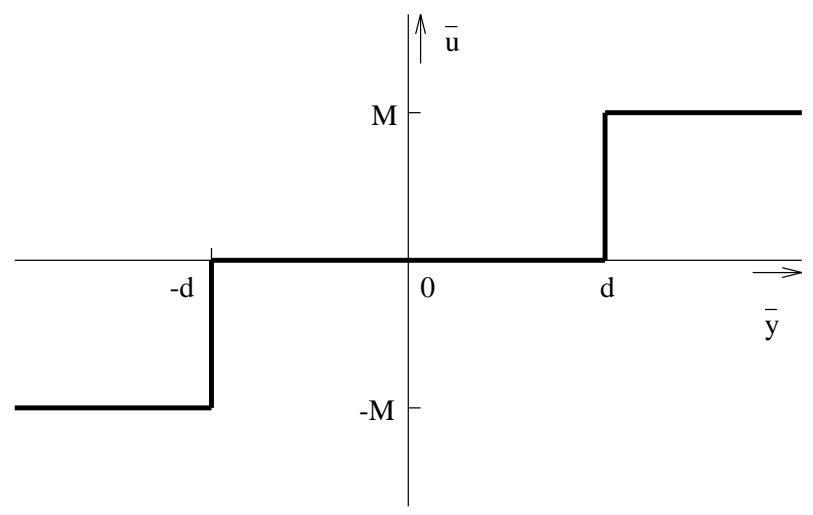

Figure 4. Relay with deadzone.

together with an algebraic constraint

$$
\bar{y}=\xi_{1}-\xi_{2}+\eta \text {. }
$$

This leads to $2^{4}=16$ modes, of which eleven are not feasible, and the remaining five correspond to the five linear parts of the characteristic given in Figure 4.

It is a far from trivial matter how to define the complete dynamics of a complementary-slackness system. So far, we have defined the modes of a complementary-slackness system, together with the additional feasibility conditions (2.5), but one also needs a rule to determine switches from one mode to another. Such switches may in general be due to external causes but may also be necessitated by the dynamics of the system itself, namely when the dynamics of a given mode would cause the inequality constraints corresponding to that mode to be violated. Indeed, a main point of our paper [16] is that such a rule can be given, at least for linear and Hamiltonian complementary-slackness systems, thus avoiding the necessity of providing transition rules on a case by case basis. We briefly describe here the proposal of [16] (see the cited paper for full details). Associated to every mode $I$ there is a consistent manifold $\mathcal{V}_{I} \subset \mathbb{R}^{N}$, consisting of all points $z$ such that there exists (for $t$ small) a solution $z_{I}(t), z_{I}(0)=z_{0}$ of the DAE (2.4). For simplicity we assume that all modes are autonomous, so that $z_{I}(t)$ is unique. Let us now consider a point $z \in \mathcal{V}_{I}$, for some $I$, and consider $z_{I}(t)$. If $z_{I}(t)$ satisfies the feasibility conditions (2.5) on some time interval $[0, \epsilon)$, then smooth continuation is possible from $z$ in mode $I$. If this does not hold a switch has to occur to another mode $J$. It was suggested in [16] to determine this new index set by the rule $J:=\left(I \backslash \Gamma_{2}(z ; I)\right) \cup \Gamma_{1}(z ; I)$, where

$$
\begin{aligned}
& \Gamma_{1}(z ; I):=\left\{i \in K \backslash I \mid \exists \epsilon>0 \text { s.t. } H_{1}\left(z_{I}(t)\right)_{i}<0, t \in(0, \epsilon)\right\} \\
& \Gamma_{2}(z ; I):=\left\{i \in I \mid \exists \epsilon>0 \text { s.t. } H_{2}\left(z_{I}(t)\right)_{i}<0, t \in(0, \epsilon)\right\} .
\end{aligned}
$$

The initial condition $z$, however, need not be in the consistent manifold $\mathcal{V}_{J}$; consequently we have to re-initialize the system to a new state $z^{\prime} \in \mathcal{V}_{J}$. The idea of [16] is that we should be able to define a foliation of $\mathbb{R}^{N}$ which is complementary to $\mathcal{V}_{J}$, and which admits a unique projection (along this foliation) from any point $z \in \mathbb{R}^{N}$ to a point $z^{\prime} \in \mathcal{V}_{J}$. It has been shown that such a foliation can be naturally given for linear complementary-slackness systems as well as (leaving out some details) for Hamiltonian complementary-slackness systems. For the newly obtained point $z^{\prime} \in \mathcal{V}_{J}$ we have to repeat the same procedure again, resulting possibly in multiple jumps at the same time instant.

Fundamental questions concerning any dynamical system are the existence of solutions, and the uniqueness of solutions. Such questions are not only basic to establishing a theoretical framework, 
but also for simulation and control. Clearly these issues are much more delicate for hybrid systems than for "ordinary" continuous-time systems. A notion of well-posedness for complementary-slackness systems has been proposed in [16] and has been shown to hold for bimodal (i.e. $k=1$ ) Hamiltonian complementary-slackness systems and linear complementary-slackness systems with positive leading Markov parameter. Uniqueness of smooth continuations of complementary-slackness systems with multiple constraints $(k>1)$ is the main topic of the present paper.

\section{The MOdE SELECTION PROBLEM}

In the simulation of hybrid systems, the following phases can be distinguished.

- Simulation of continuous motion within a given mode. In principle this is not different from the simulation of continuous dynamical systems, since we are concerned here with the situation in which the system is in a fixed mode; however, in the multimodal context the equations of motion will more often than not be given by differential and algebraic equations (cf. the example in the Introduction). Of course, there is an extensive literature on DAE's (see for instance [1]).

- Event detection. Events are usually categorized as state events (triggered by state variables that cross certain thresholds) and time events (triggered from outside, at certain given times). The detection of state events is a nontrivial matter in particular when a DAE solver is being used to carry out the continuous simulation.

- Mode selection. When an event takes place, there will in general be a change of mode, and so one is confronted with the problem of determining which mode should be chosen. In some cases, in particular in connection with time events, the new mode may be prescribed as part of the event (for instance gear shift in a car). However in other cases the choice of the new mode may depend on the continuous state and then the challenge is to construct a mapping from the continuous state to the discrete state, or in other words to define a labeled partitioning of the continuous state space.

- Re-initialization. As is already clear in the example discussed in the Introduction, in some cases events may be accompanied by jumps in the continuous state variable. Again in some cases the jump may be determined exogenously but in other cases will have to be computed on the basis of certain rules. A projection rule was suggested in [16] for linear and Hamiltonian systems within a complementarity framework.

Here we shall concentrate on the uniqueness of smooth continuations and the mode selection problem. We shall therefore work under the assumption that an initial continuous state is given from which at least one smooth continuation is possible, so no re-initialization is necessary. Moreover, we shall assume that there are no external inputs (otherwise the problem has to be reformulated as uniqueness of smooth continuations for a given external input-we believe that this is a fairly straightforward generalization).

As already shown in [16] it is not difficult to find examples of complementary-slackness systems that exhibit nonuniqueness of smooth continuations. For a simple example of this phenomenon, consider an integrator

$$
\dot{x}(t)=u(t), \quad y(t)=x(t)
$$

in closed-loop with a relay

$$
\begin{array}{ll}
u(t)=1 & \text { if } y(t) \geq 0 \\
-1 \leq u(t) \leq 1 & \text { if } y(t)=0 \\
u(t)=-1 & \text { if } y(t) \leq 0 .
\end{array}
$$


It was shown in the previous section that such a system can be modelled as a complementary-slackness system. Note that from the initial state $x(0)=0$, there are three possible smooth continuations: (i) $x(t)=0, y(t)=0, u(t)=0$, (ii) $x(t)=t, y(t)=t, u(t)=1$, (iii) $x(t)=-t, y(t)=-t, u(t)=-1$. So the above closed-loop is not well-posed as a dynamical system. If the sign of the feedback coupling is reversed, however, there is only one smooth continuation from each initial state.

The mode selection problem is of course related to the problem of uniqueness of smooth continuations; under our standing assumptions, nonuniqueness of smooth continuations can only arise from the existence of multiple solutions to the mode selection problem. But even when we do have uniqueness of smooth continuations, it is often a nontrivial problem to determine which mode provides this smooth continuation for a given continuous state. To illustrate this, consider the example of the Introduction and assume that we are at an initial state $x_{0}=\left(x_{10}, x_{20}, x_{30}, x_{40}\right)$ with $x_{10}=0$ and $x_{20}=0$; also assume that the input $v(t)$ is zero. In this situation, smooth continuation is possible, and the mode selection problem has the following solution:

- continuation in constrained mode if $x_{30}<0$, or $x_{30}=0$ and $x_{40}<0$;

- continuation in unconstrained mode if $x_{30}>0$, or $x_{30}=0$ and $x_{40}>0$.

These rules leave open one case, namely when $x_{30}=0$ and $x_{40}=0$. Together with the assumptions $x_{10}=0$ and $x_{20}=0$, these conditions imply that the system is at rest, and the constrained and unconstrained modes agree in that case. Below we shall discuss how to derive these rules (which appear to be consistent with physical reality, at least in the example) within the complementarity formalism.

We start with a rather general nonlinear (although still semi-explicit) setting, in which equations of motion are given by

$$
\begin{aligned}
\dot{x}(t) & =f(x(t), u(t)) \\
y(t) & =h(x(t), u(t))
\end{aligned}
$$

together with the complementarity conditions

$$
y(t) \geq 0, \quad u(t) \geq 0, \quad y(t)^{T} u(t)=0 .
$$

As noted above we consider for simplicity the case in which there are no external inputs. The symbol $u(t)$ in the above equations should therefore be viewed as a (generalized) 'constraint force', or in system-theoretic terms as an implicitly defined output, although it is written as an input function (and we shall actually use some of the theory of input/output systems). Explicitly, we shall assume the following.

Assumption 3.1 The functions $f$ and $h$ defining the dynamics of (3.1) are real-analytic.

Assumption 3.2 For each index set $I \subset\{1, \ldots, k\}$ the equations (3.1) together with the equality constraints

$$
y_{i}(t)=0 \quad(i \in I), \quad u_{i}(t)=0 \quad(i \notin I)
$$

form a system of DAE's with the following properties. The set of all vectors $x_{0}$ for which there exists a solution $(x(\cdot), y(\cdot), u(\cdot))$ of (3.1)-(3.3) such that $x\left(t_{0}\right)=x_{0}$ for some time instant $t_{0}$ forms a smooth manifold $\mathcal{V}_{I}$, and for each $x_{0}$ and each $t_{0}$ there is only one solution $(x(\cdot), y(\cdot), u(\cdot))$ of $(3.1)-(3.3)$ such that $x\left(t_{0}\right)=x_{0}$. 
Assumption 3.1 could be weakened for parts of the development below, but we shall not bother to give specific smoothness conditions in each case. The condition of Assumption 3.2 comes down to requiring that the system of DAE's can be reduced to an ordinary differential equation on the consistent manifold. The transformation from DAE to ODE can be carried out by so-called index reduction algorithms (see for instance [6]); such algorithms also provide sufficient conditions for Assumption 3.2 to hold.

The problem with solving (3.1) together with the inequality constraints (3.2) is that one has to determine which index set $I$ has the property that the solution of (3.1)-(3.3) coincides with that of (3.1)-(3.2). One option is simply to try all possibilities-solve (3.1) together with (3.3) for some chosen candidate index set $I$, and see whether the computed solution is such that the inequality constraints $y(t) \geq 0$ and $u(t) \geq 0$ are satisfied on some interval $[0, \varepsilon)$. Under the assumption that smooth continuation is possible from $x_{0}$, there must at least be one index set for which the constraints will indeed be satisfied, but finding that index set in this way requires in the worst case the integration of $2^{k}$ systems of $n+k$ differential/algebraic equations in $n+k$ unknowns.

In order to try an alternative approach which leads to an algebraic problem formulation, let us note first that we can derive from (3.1) a number of relations between the successive time derivatives of $y(\cdot)$, evaluated at $t=0$, and the same quantities derived from $u(\cdot)$. By differentiating the second line of (3.1) and using the first line, we get

$$
\begin{aligned}
y(t) & =h(x(t), u(t)) \\
\dot{y}(t) & =\frac{\partial h}{\partial x}(x(t), u(t)) f(x(t), u(t))+\frac{\partial h}{\partial u}(x(t), u(t)) \dot{u}(t) \\
& =: \quad F_{1}(x(t), u(t), \dot{u}(t)),
\end{aligned}
$$

and in general

$$
y^{(j)}(t)=F_{j}\left(x(t), u(t), \ldots, u^{(j)}(t)\right)
$$

where $F_{j}$ is a function that can be specified explicitly in terms of $f$ and $h$. From this we obviously get the relations

$$
\begin{aligned}
y(0) & =h\left(x_{0}, u(0)\right)=: F_{0}\left(x_{0}, u(0)\right) \\
\dot{y}(0) & =F_{1}\left(x_{0}, u(0), \dot{u}(0)\right) \\
& \vdots \\
y^{(j)}(0) & =F_{j}\left(x_{0}, u(0), \ldots, u^{(j)}(0)\right) \\
& \vdots
\end{aligned}
$$

From the complementarity conditions (3.2), it follows moreover that for each index $i$ either

$$
\left(y_{i}(0), \dot{y}_{i}(0), \ldots\right)=0 \text { and }\left(u_{i}(0), \dot{u}_{i}(0), \ldots\right) \succeq 0
$$

or

$$
\left(y_{i}(0), \dot{y}_{i}(0), \ldots\right) \succeq 0 \text { and }\left(u_{i}(0), \dot{u}_{i}(0), \ldots\right)=0
$$

(or both), where we use the symbol $\succeq$ to denote lexicographic nonnegativity. (A sequence $\left(a_{0}, a_{1}, \ldots\right.$ ) of real numbers is said to be lexicographically nonnegative if either all $a_{i}$ are zero or the first nonzero element is positive.) This suggests the formulation of the following problem. 
Problem DCP $(\ell)$. Given smooth functions $F_{j}: \mathbb{R}^{n+(j+1) k} \rightarrow \mathbb{R}^{k}(j=0, \ldots, \ell)$ that are constructed from smooth functions $f: \mathbb{R}^{n} \rightarrow \mathbb{R}^{n}$ and $h: \mathbb{R}^{n} \rightarrow \mathbb{R}^{k}$ via (3.4), find, for given $x_{0} \in \mathbb{R}^{n}$, sequences $\left(y^{0}, \ldots, y^{\ell}\right)$ and $\left(u^{0}, \ldots, u^{\ell}\right)$ of $k$-vectors such that for $j=0, \ldots, \ell$ we have

$$
y^{j}=F_{j}\left(x_{0}, u^{0}, \ldots, u^{j}\right)
$$

and for each index $i \in\{1, \ldots, k\}$ at least one of the following is true:

$$
\begin{array}{ll}
\left(y_{i}^{0}, y_{i}^{1}, \ldots, y_{i}^{\ell}\right)=0 \quad \text { and } \quad\left(u_{i}^{0}, u_{i}^{1}, \ldots, u_{i}^{\ell}\right) \succeq 0 \\
\left(y_{i}^{0}, y_{i}^{1}, \ldots, y_{i}^{\ell}\right) \succeq 0 \quad \text { and } \quad\left(u_{i}^{0}, u_{i}^{1}, \ldots, u_{i}^{\ell}\right)=0 .
\end{array}
$$

The set of indices for which (3.9) holds and (3.10) does not will be called the active index set, and the set of indices for which (3.10) is true and (3.9) is not satisfied will be called the inactive index set (as determined on the basis of $\ell$ steps, and corresponding to a given solution). Even if the solution to DCP is unique, there may be indices that are not classified as active or inactive; for these indices one has $y_{i}^{j}=0$ as well as $u_{i}^{j}=0$ for $j=0, \ldots, \ell$. By increasing $\ell$ if necessary, one may arrive at a situation in which all indices are classified as active or inactive. Since the active index set determines the 'mode' or 'discrete state' of the system, we have then constructed a discrete state $I\left(x_{0}\right)$ corresponding to the continuous state $x_{0}$. It may also happen that the discrete state $I\left(x_{0}\right)$ is not uniquely determined; in this case one may still have uniqueness of solutions, namely when all modes compatible with $x_{0}$ produce the same trajectory starting from $x_{0}$.

The problem DCP is a generalization of the nonlinear complementarity problem, which can be formulated as follows (in a parametrized form).

Problem NCP. Given a smooth function $F: \mathbb{R}^{n+k} \rightarrow \mathbb{R}^{k}$, find, for given $x \in \mathbb{R}^{n}, k$-vectors $y$ and $u$ such that

$$
y=F(x, u)
$$

and for each index $i \in\{1, \ldots, k\}$ at least one of the following is true:

$$
\begin{array}{llll}
y_{i}=0 & \text { and } & & u_{i} \geq 0 \\
y_{i} \geq 0 & \text { and } & & u_{i}=0 .
\end{array}
$$

An equivalent (and in fact more standard) formulation of the second condition is: $y \geq 0, u \geq 0$ (where the inequalities are interpreted componentwise), and $y^{T} u=0$. Because of the similarity of the two problems we shall refer to Problem DCP as a dynamic complementarity problem. The terminology seems so natural that we use it in spite of the fact that a related but different problem has already been termed a dynamic complementarity problem by Chen and Mandelbaum [2].

Computational methods for the NCP form a highly active research subject (see [7] for a survey), due to the many applications in particular in equilibrium programming. The DCP is a generalized and parametrized form of the NCP and given the fact that the latter problem is already considered a major computational challenge, one may wonder whether the approach taken in the previous paragraphs can be viewed as promising from a computational point of view. Fortunately, it turns out that under fairly mild assumptions the DCP can be reduced to a series of linear complementarity problems. The linear complementarity problem (LCP) can be formulated as follows.

Problem LCP. Given a vector $q \in \mathbb{R}^{k}$ and a matrix $M \in \mathbb{R}^{k \times k}$, find $k$-vectors $y$ and $u$ such that

$$
y=q+M u, \quad y \geq 0, \quad u \geq 0, \quad y^{T} u=0 .
$$

The LCP has been studied extensively, in particular because of its applications in game theory and mathematical programming. A wealth of theoretical results and computational methods has been collected in the volume [3] by Cottle, Pang, and Stone. 
We shall now discuss the assumptions under which the reduction to a sequence of LCP's is possible. Firstly, we assume that the dynamics (3.1) can be written in the affine form

$$
\begin{aligned}
\dot{x}(t) & =f(x(t))+\sum_{i=1}^{k} g_{i}(x(t)) u_{i}(t) \\
y(t) & =h(x(t)) .
\end{aligned}
$$

Such a system is sometimes known as a smooth affine nonlinear control system with outputs (see for instance [12]); as before however we note that under the complementarity conditions (3.2) and under our standing assumptions the functions $u_{i}(t)$ are implicitly defined outputs rather than control inputs. For a system in the above form, the first derivative of $y(\cdot)$ can be computed as

$$
\begin{aligned}
\dot{y}(t) & =\frac{\partial h}{\partial x}(x(t)) f(x(t))+\sum_{i=1}^{k} \frac{\partial h}{\partial x}(x(t)) g_{i}(x(t)) u_{i}(t) \\
& =: \quad L_{f} h(x(t))+\sum_{i=1}^{k} L_{g_{i}} h(x(t)) u_{i}(t) .
\end{aligned}
$$

(The notation in the second line uses Lie derivatives, see for instance [14, p. 207].) It may happen that all functions $L_{g_{i}} h(x)$ vanish, so that $u$ actually does not appear in the expression for $\dot{y}$. In that case the second time derivative of $y(\cdot)$ can be computed as

$$
\ddot{y}(t)=L_{f}^{2} h(x(t))+\sum_{i=1}^{k} L_{g_{i}} L_{f} h(x(t)) u_{i}(t) .
$$

Again it may happen that all functions $L_{g_{i}} L_{f} h(x)$ vanish; then an expression for the third derivative of $y(\cdot)$ can be written down which will depend on $u(\cdot)$ via the functions $L_{g_{i}} L_{f}^{2} h(x)$. We now introduce the following assumption.

Assumption 3.3 The functions $f, g_{i}$, and $h$ appearing in (3.15) enjoy the following property. There exists an integer $\rho \geq 1$ such that for $r=0, \ldots, \rho-2$ the functions $L_{g_{i}} L_{f}^{r} h(x)$ vanish identically, whereas the $k \times k$ matrix

$$
L_{g} L_{f}^{\rho-1} h(x):=\left[L_{g_{1}} L_{f}^{\rho-1} h(x) \cdots L_{g_{k}} L_{f}^{\rho-1} h(x)\right]
$$

is nonsingular for all $x$.

In the nonlinear control literature, the above assumption is known as the assumption of uniform relative degree and nonsingular decoupling matrix (see for instance [12, Ch.8]). In particular the integer $\rho$ is known as the (uniform) relative degree of the system (3.15), whereas the matrix appearing in (3.18) is called the decoupling matrix. A slightly different convention was used in [12] where $\rho-1$ is called the relative degree. For the purposes of a local analysis, the assumption of nonsingularity of the decoupling matrix for all $x$ could be replaced by nonsingularity for all points $x$ in a neighborhood of a given point $x_{0}$.

We also need to introduce also some terminology from matrix theory. Given a matrix $M$ of size $k \times k$ and two nonempty subsets $I$ and $J$ of $\{1, \ldots, k\}$ of equal cardinality, the $(I, J)$-minor of $M$ is the determinant of the square submatrix $M_{I J}:=\left(m_{i j}\right)_{i \in I, j \in J}$. The $(I, I)$-minors are also known as the principal minors; so a $k \times k$-matrix has $2^{k}-1$ principal minors. See for instance [5, p. 2] for this terminology.

The proof of the theorem below is based on reduction of the DCP for affine systems, under suitable assumptions, to a series of LCP's. The idea of this reduction is due to Lötstedt [11], who applied it in the context of mechanical systems with inequality constraints.

THEOREM 3.4 Consider the system of equations (3.15) together with the complementarity conditions (3.2), and suppose that assumptions 3.1, 3.2, and 3.3 are satisfied, with uniform relative degree $\rho$. Let $x_{0} \in \mathbb{R}^{n}$ be such that

$$
h\left(x_{0}\right) \geq 0, \cdots, L_{f}^{\rho-1} h\left(x_{0}\right) \geq 0 .
$$


If all principal minors of the decoupling matrix $L_{g} L_{f}^{\rho-1} h\left(x_{0}\right)$ at $x_{0}$ are positive, then the dynamic complementarity problem DCP( $\ell)$ has for each $\ell$ a solution $\left(\left(y^{0}, \ldots, y^{\ell}\right),\left(u^{0}, \ldots, u^{\ell}\right)\right)$; moreover this solution is unique except for the values of $u_{i}^{j}$ for $j>\ell-\rho$ and indices $i$ such that $y_{i}^{j}=0$ for all $j=0, \ldots, \ell$.

Proof It follows from the special form of (3.15) and the assumption on the relative degree that the equations of the DCP will take the following form, in which the $\phi_{j}$ 's denote functions that can be computed explicitly from the given functions $f, g_{i}$, and $h$ :

$$
\begin{aligned}
y^{0} & =h\left(x_{0}\right) \\
& \vdots \\
y^{\rho-1} & =L_{f}^{\rho-1} h\left(x_{0}\right) \\
y^{\rho} & =\phi_{0}\left(x_{0}\right)+L_{g} L_{f}^{\rho-1} h\left(x_{0}\right) u^{0} \\
& \vdots \\
y^{\rho+j} & =\phi_{j}\left(x_{0}, u^{0}, \ldots, u^{j-1}\right)+L_{g} L_{f}^{\rho-1} h\left(x_{0}\right) u^{j}
\end{aligned}
$$

From this and (3.19) it is already obvious that the claim of the theorem holds for $\ell=0, \ldots, \rho-1$. We now continue by induction and so we shall carry out the proof assuming that $\ell \geq \rho$ and that the claim in the theorem holds for $\operatorname{DCP}(\ell-1)$. Let $I_{\ell-1}$ and $J_{\ell-1}$ denote the active index set and the inactive index set, respectively, as determined by $\operatorname{DCP}(\ell-1)$, and let $K_{\ell-1}$ denote the set of remaining indices, whose status is not determined by $\operatorname{DCP}(\ell-1)$. A solution $\left(\left(y^{0}, \ldots, y^{\ell}\right),\left(u^{0}, \ldots, u^{\ell}\right)\right)$ of $\operatorname{DCP}(\ell)$ can now be constructed as follows. The components $y^{j}$ and $u^{j}$ for $j=0, \ldots, \ell-1$ are taken from the solution for $\operatorname{DCP}(\ell-1)$; by this choice one satisfies automatically all equations of $\operatorname{DCP}(\ell)$ except for the last one, which is

$$
y^{\ell}=\phi_{\ell-\rho}\left(x_{0}, u^{0}, \ldots, u^{\ell-\rho-1}\right)+L_{g} L_{f}^{\rho-1} h\left(x_{0}\right) u^{\ell-\rho}
$$

which we write in abbreviated form as

$$
y^{\ell}=z^{\ell}+D u^{\ell-\rho} .
$$

Note that the vector $z^{\ell}$ depends only on the components of the solution of $\operatorname{DCP}(\ell-1)$ that are uniquely determined; the matrix $D$ is just the decoupling matrix at $x_{0}$. In addition to (3.22), the complementarity conditions of $\operatorname{DCP}(\ell)$ have to be satisfied; after eliminating all conditions that are satisfied automatically by building the solution from the one that was obtained from $\operatorname{DCP}(\ell-1)$, this leaves us with the conditions

$$
y_{i}^{\ell}=0 \quad\left(i \in I_{\ell-1}\right), \quad u_{i}^{\ell-\rho}=0 \quad\left(i \in J_{\ell-1}\right)
$$

and

$$
y_{i}^{\ell} \geq 0, \quad u_{i}^{\ell-\rho} \geq 0, \quad y_{i}^{\ell} u_{i}^{\ell-\rho}=0 \quad\left(i \in K_{\ell-1}\right) .
$$

Dividing up the equation (3.22) in three parts corresponding to the index sets $J_{\ell-1}, I_{\ell-1}$, and $K_{\ell-1}$, and dropping all indices and subindices that depend on $\ell$ to alleviate the notational burden, we get

$$
\left[\begin{array}{c}
y_{I} \\
y_{J} \\
y_{K}
\end{array}\right]=\left[\begin{array}{c}
z_{I} \\
z_{J} \\
z_{K}
\end{array}\right]+\left[\begin{array}{ccc}
D_{I I} & D_{I J} & D_{I K} \\
D_{J I} & D_{J J} & D_{J K} \\
D_{K I} & D_{K J} & D_{K K}
\end{array}\right]\left[\begin{array}{c}
u_{I} \\
u_{J} \\
u_{K}
\end{array}\right]
$$


By (3.23), we have to take $y_{I}=0$ and $u_{J}=0$. We see that the remaining components have to be chosen such that the following equations are satisfied:

$$
\begin{aligned}
0 & =z_{I}+D_{I I} u_{I}+D_{I K} u_{K} \\
y_{J} & =z_{J}+D_{J I} u_{I}+D_{J K} u_{K} \\
y_{K} & =z_{K}+D_{K I} u_{I}+D_{K K} u_{K} .
\end{aligned}
$$

Moreover, the complementarity conditions that follow from (3.24) must hold:

$$
y_{K} \geq 0, \quad u_{K} \geq 0, \quad y_{K}^{T} u_{K}=0
$$

By assumption the determinant of $D_{I I}$ is positive and hence nonzero, so that $u_{I}$ can be solved in terms of $z_{I}$ and $u_{K}$ from (3.25). Inserting the result in (3.27) leads to the equation

$$
y_{K}=z_{K}+D_{K I} D_{I I}^{-1} z_{I}+\left(D_{K K}-D_{K I} D_{I I}^{-1} D_{I K}\right) u_{K}
$$

The above equation together with the complementary conditions (3.28) constitutes a standard LCP. From our assumption that all principal minors of $D$ are positive, it follows [15] that the same property is true for $D_{K K}-D_{K I} D_{I I}^{-1} D_{I K}$, since this matrix is a Schur complement of a principal submatrix of $D$. From the general theory of the linear complementary problem (see for instance [3, Thm.3.3.7]), it then follows that the LCP (3.28)-(3.29) has a unique solution. This determines $y_{K}$ and $u_{K}$; then finally $u_{I}$ and $y_{J}$ follow from (3.25) and (3.26). In this way we have constructed a solution of $\mathrm{DCP}(\ell)$. The uniqueness claim follows from an inspection of the above construction method.

Solving the problem $\operatorname{DCP}(\ell)$ in principle gives more information as $\ell$ is increased, but in many cases the information obtained after just a few differentiations will be enough to determine a mode uniquely. In principle though it seems difficult to give an upper bound on the number of steps that would be required to obtain complete information; for the special case of linear systems however the situation is different, as we will see below. The theorem above is nevertheless strong enough to provide sufficient conditions for uniqueness of smooth continuations.

THEOREM 3.5 Consider the system of equations (3.15) together with the complementarity conditions (3.2). Suppose that assumptions 3.1, 3.2, and 3.3 are satisfied, with uniform relative degree $\rho$. If all principal minors of the decoupling matrix $L_{g} L_{f}^{\rho-1} h\left(x_{0}\right)$ at a given $x_{0} \in \mathbb{R}^{n}$ are positive, and smooth continuation from $x_{0}$ is possible, then this smooth continuation is unique.

Proof First of all, note that smooth continuations within a given mode are unique by Assumption 3.2. Solutions according to mode $I$ will be denoted by $(x(\cdot ; I), y(\cdot ; I), u(\cdot ; I))$, where it is understood that $x(0 ; I)=x_{0}$. Consider now two solutions of (3.15)-(3.2) corresponding to different modes $I_{1}$ and $I_{2}$. The sequences $\left(y^{(j)}\left(0 ; I_{1}\right), u^{(j)}\left(0 ; I_{1}\right)\right)$ and $\left(y^{(j)}\left(0 ; I_{2}\right), u^{(j)}\left(0 ; I_{2}\right)\right)$ must then both satisfy $\operatorname{DCP}(\ell)$ for all values of $\ell$. By the uniqueness property of solutions to $\operatorname{DCP}(\ell)$, it follows that $y^{(j)}\left(0 ; I_{1}\right)=y^{(j)}\left(0 ; I_{2}\right)$ for all $j$, and likewise for the $u$-variables. Since the solutions are real-analytic as a consequence of Assumption 3.1, it follows that actually $y\left(\cdot ; I_{1}\right)=y\left(\cdot ; I_{2}\right)$ and $u\left(\cdot ; I_{1}\right)=u\left(\cdot ; I_{2}\right)$. This implies that the solution $\left(x\left(\cdot ; I_{2}\right), y\left(\cdot ; I_{2}\right), u\left(\cdot ; I_{2}\right)\right)$ also satisfies the conditions of mode $I_{1}$. Uniqueness of the solutions now follows from Assumption 3.2.

REMARK 3.6 Note that in the above theorem there is no claim that the mode associated with the initial condition $x_{0}$ is uniquely determined. In the example of the Introduction, the initial state $x_{0}=0$ gives rise to the solution $x(t)=0$ which belongs to the constrained mode as well as to the unconstrained mode. Because the active and inactive index sets $I_{\ell}$ and $J_{\ell}$ determined by $\operatorname{DCP}(\ell)$ are nondecreasing functions taking values in a finite lattice, there must exist an $\ell^{*}$ such that $I_{\ell}=I_{\ell^{*}}$ and $J_{\ell}=J_{\ell^{*}}$ for all $\ell \geq \ell^{*}$. Of course we then also have $K_{\ell}=K_{\ell^{*}}$ for $\ell \geq \ell^{*}$. The unique smooth continuation starting from the given initial condition $x_{0}$ will take place according to any mode $I$ such 
that $I_{\ell^{*}} \subset I \subset I_{\ell^{*}} \cup K_{\ell^{*}}$ (all such modes give rise to the same solution starting from $x_{0}$ ). Although the initial conditions for which such an indeterminacy occurs will be relatively rare, one might for the purposes of completeness think of classifying indices corresponding to constraints not just as active or inactive, but as active, inactive, or indeterminate. This would give rise to a description with $3^{k}$ rather than $2^{k}$ discrete states.

REMARK 3.7 In the formulation of the system equations (3.15) we have not included an affine feedthrough term; this follows common practice in nonlinear control theory, where $y(t)$ may indicate coordinates on a manifold so that an additive structure is not available. Nevertheless it may be meaningful in some cases to write down equations in the form

$$
\begin{aligned}
& \dot{x}(t)=f(x(t))+\sum_{i=1}^{k} g_{i}(x(t)) u_{i}(t) \\
& y(t)=h(x(t))+\sum_{i=1}^{k} r_{i}(x(t)) u_{i}(t) .
\end{aligned}
$$

If the matrix $R(x):=\left[r_{1}(x) \cdots r_{k}(x)\right]$ has positive principal minors at $x=x_{0}$, then a similar reasoning as in the theorems above can be used to prove the uniqueness of smooth continuations.

\section{Some Particular CASES}

In this section we shall consider a number of special situations in which the results of the previous section can be applied or extended. We shall consider Hamiltonian systems, passive systems, and linear systems.

\subsection{Hamiltonian systems}

Consider the Hamiltonian complementary-slackness system (2.10), with real-analytic Hamiltonian (total energy)

$$
H(q, p)=\frac{1}{2} p^{T} M^{-1}(q) p+V(q), \quad M(q)=M^{T}(q)>0
$$

where $M(q)$ is the generalized mass matrix. Assume that the geometric inequality constraints $C_{i}(q) \geq$ 0 are real-analytic and independent, that is

$$
\operatorname{rank} \frac{\partial C^{T}}{\partial q}(q)=k, \quad \text { for all } q \text { with } C(q) \geq 0 .
$$

It is immediately seen that the system has uniform relative degree 2 with decoupling matrix $D$ given as

$$
D(q)=\left[\frac{\partial C^{T}}{\partial q}(q)\right]^{T} M^{-1}(q) \frac{\partial C^{T}}{\partial q}(q) .
$$

Since $M(q)>0$ also $M^{-1}(q)>0$, and hence from (4.2) it follows that

$$
D(q)=D^{T}(q)>0, \quad \text { for all } q \text { with } C(q) \geq 0 .
$$

A positive definite matrix has the property that all its principal minors are positive, and hence if we start from a point $x_{0}=\left(q_{0}, p_{0}\right)$ such that

$$
C\left(q_{0}\right) \geq 0, \quad\left[\frac{\partial C^{T}}{\partial q}\left(q_{0}\right)\right]^{T} M^{-1}\left(q_{0}\right) p_{0} \geq 0
$$

then the dynamic complementarity problem $\operatorname{DCP}(\ell)$ has for each $\ell$ a solution $\left(\left(y^{0}, \cdots, y^{\ell}\right),\left(u^{0}, \cdots, u^{\ell}\right)\right)$, which is unique except for the values of $u^{j}$ with $j>\ell-\rho$. Consequently we also have uniqueness of smooth continuations. Note that these results are unchanged if we replace the second set of equations in (2.10) by (2.11) (involving dissipation). 


\subsection{Passive systems}

A system (3.15) is called passive (see [17]) if there exists a function $V(x) \geq 0$ (a storage function) such that

$$
\begin{aligned}
& L_{f} V(x) \leq 0 \\
& L_{g_{i}} V(x)=h_{i}(x), \quad i=1, \cdots, k .
\end{aligned}
$$

Let us assume the following non-degeneracy condition on the storage function $V$ :

$$
\operatorname{rank}\left[L_{g_{j}} L_{g_{i}} V(x)\right]_{i, j=1, \cdots, k}=k, \text { for all } x \text { with } h(x) \geq 0 .
$$

Since $L_{g_{j}} h_{i}=L_{g_{j}} L_{g_{i}} V$ it follows that a passive system satisfying (4.7) has uniform relative degree 1, with decoupling matrix $D(x)$ given by the matrix in (4.7). If we additionally assume that the input vector fields $g_{1}, \cdots, g_{k}$ are commuting, i.e.,

$$
\left[g_{i}, g_{j}\right]=0, \quad i, j \in\{1, \cdots, k\}
$$

then this decoupling matrix is symmetric, since (see e.g. [12, Ch. 2])

$$
L_{g_{i}} L_{g_{j}} V(x)=L_{g_{j}} L_{g_{i}} V(x)-L_{\left[g_{i}, g_{j}\right]} V(x) .
$$

Hence $D(x)=D^{T}(x) \geq 0$, and by (4.7) $D(x)=D^{T}(x)>0$. Therefore, as in the previous subsection, the dynamic complementarity problem is solvable for each $\ell$.

An example is provided by a passive (linear) electrical network with diodes, as briefly discussed in Section 2. If the storage function, which in this case can be taken to be total energy stored in the network (e.g. at the capacitors and inductors) is positive definite, and the matrix $B$ in (2.8) has full column rank, then the above properties hold.

\subsection{Linear systems}

In this subsection we shall consider the case in which we have linear dynamics in (3.15). We shall moreover allow a feedthrough term which we shall denote by $D$ following the usual conventions of linear system theory; so in this subsection $D$ does not in general indicate a decoupling matrix. The equations (3.15) are thus replaced by

$$
\begin{aligned}
\dot{x}(t) & =A x(t)+B u(t) \\
y(t) & =C x(t)+D u(t) .
\end{aligned}
$$

Linear complementarity modeling applies for instance to electrical networks with linear elements and diodes, to certain mechanical systems made up of masses and linear springs (or rotational inertias and corresponding elasticity), and to the Hamiltonian equations for linear-quadratic optimal control problems with linear inequality constraints.

For linear systems the results of the previous section can be sharpened considerably. First of all, the DCP can be specialized to the linear dynamic complementarity problem shown below; note that in this problem the number of steps is not taken as a variable but rather is determined by the problem data.

Problem LDCP. Given matrices $A, B, C, D$ of sizes $n \times n, n \times k, k \times n$, and $k \times k$ respectively, find, for given $x_{0} \in \mathbb{R}^{n}$, sequences $\left(y^{0}, y^{1}, \ldots, y^{n-1}\right)$ and $\left(u^{0}, u^{1}, \ldots, u^{n-1}\right)$ of $k$-vectors such that the following equations hold:

$$
\begin{aligned}
y^{0} & =C x_{0}+D u^{0} \\
y^{1} & =C A x_{0}+C B u^{0}+D u^{1} \\
& \vdots \\
y^{n-1} & =C A^{n-1} x_{0}+C A^{n-2} B u^{0}+\cdots+C B u^{n-2}+D u^{n-1}
\end{aligned}
$$


and for all indices $i \in\{1, \ldots, k\}$ at least one of the following is true:

$$
\begin{aligned}
& \left(y_{i}^{0}, y_{i}^{1}, \ldots, y_{i}^{n-1}\right)=0 \text { and }\left(u_{i}^{0}, u_{i}^{1}, \ldots, u_{i}^{n-1}\right) \succeq 0 \\
& \left(y_{i}^{0}, y_{i}^{1}, \ldots, y_{i}^{n-1}\right) \succeq 0 \text { and }\left(u_{i}^{0}, u_{i}^{1}, \ldots, u_{i}^{n-1}\right)=0 .
\end{aligned}
$$

The restriction to $n-1$ steps is motivated by the theorem below. Note that a system of the form (4.10) has uniform relative degree $\rho \geq 1$ with invertible decoupling matrix if $D=0$ and $C A^{j} B=0$ for $j=0, \ldots, \rho-2$, and moreover $C A^{\rho-1} B$ is invertible. When $D$ is nonsingular, we shall say that the system has uniform relative degree 0 with invertible decoupling matrix. The matrices $M_{0}:=D$, $M_{j}:=C A^{j-1} B$ are also known as the Markov parameters of the system (4.10). The condition of uniform relative degree $\rho$ and invertible decoupling matrix is equivalent to the condition that the Smith-McMillan form at infinity of the transfer matrix $G(s):=C(s I-A)^{-1} B+D$ (see for instance [8, p. 415] or [10, p. 17]) is $s^{-\rho} I_{k}$ (where $I_{k}$ denotes the $k \times k$ unit matrix), or in other words that $G(s)$ has $k$ zeros at infinity, all of order $\rho$. For this reason we shall say that the system (4.10) has uniform zero structure at infinity when $M_{j}=0$ for $j=0, \ldots, \rho-1$ and $M_{\rho}$ is invertible, and $M_{\rho}$ will be called the leading Markov parameter.

THEOREM 4.1 Consider the system (4.10) together with the complementarity conditions (3.2). Assume that the system (4.10) has uniform zeros at infinity of order $\rho$, and that all principal minors of the leading Markov parameter are positive. Under these conditions the LDCP has, for each $x_{0}$ such that $C A^{j} x_{0} \geq 0(j=0, \ldots, \rho-1)$, a solution $\left(\left(y^{0}, \ldots, y^{n-1}\right),\left(u^{0}, \ldots, u^{n-1}\right)\right)$, unique except for the values of $u_{i}^{j}$ for $j \geq n-\rho$ and indices $i$ such that $y_{i}^{j}=0$ for all $j=0, \ldots, n-1$. For such initial conditions there exists an $\varepsilon>0$ such that (4.10)-(3.2) has a solution on $[0, \varepsilon)$; moreover, this solution is unique.

Proof The first claim is immediate from the nonlinear case (see also Remark 3.7). Let $I$ be the active index set as indicated by the LDCP for the given initial value $x_{0}$. Since indices $i$ such that $C_{i} A^{j} x_{0}>0$ for some $j \in\{0, \ldots, \rho-1\}$ will not belong to $I$, we have that $C_{i} A^{j} x_{0}=0$ for $i \in I$ and $j=0, \ldots, \rho-1$. Note that the motion in mode $I$ is described by the equations

$$
\begin{aligned}
\dot{x} & =A x+B_{I} u \\
0=y & =C_{I} x+D_{I I} u
\end{aligned}
$$

where $C_{I}$ denotes the submatrix of $C$ consisting of the rows with indices $i \in I$, and $B_{I}$ is the submatrix of $B$ consisting of the columns with indices $i \in I$. From the assumptions we have made, it follows that (in case $\rho=0) D_{I I}$ is invertible, or (in case $\left.\rho>0\right) D_{I I}=0, C_{I} A^{j} B_{I}=0$ for $j=0, \ldots, \rho-2$, and $C_{I} A^{\rho-1} B_{I}$ is invertible (since $\operatorname{det} C_{I} A^{\rho-1} B_{I}$ is a principal minor of $C A^{\rho-1} B$ ). This implies that the set of consistent initial conditions for mode $I$ is

$$
V_{I}=\left\{x \mid C_{I} A^{j} x=0, j=0, \ldots, \rho-1\right\} .
$$

Since we already found that $C_{i} A^{j} x_{0}=0$ for $i \in I$ and $j=0, \ldots, \rho-1$, it follows that a smooth continuation from $x_{0}$ according to the equality constraints of mode $I$ is possible. Denote the corresponding solution by $(x(\cdot), y(\cdot), u(\cdot))$. From the uniqueness property of solutions to the LDCP, it follows that for each index $i$ we have either

$$
\left(y_{i}(0), \dot{y}_{i}(0), \ldots, y_{i}^{(n-1)}(0)\right) \succeq 0 \quad \text { and } \quad\left(u_{i}(0), \dot{u}_{i}(0), \ldots, u_{i}^{(n-1)}(0)\right)=0
$$

or

$$
\left(y_{i}(0), \dot{y}_{i}(0), \ldots, y_{i}^{(n-1)}(0)\right)=0 \quad \text { and } \quad\left(u_{i}(0), \dot{u}_{i}(0), \ldots, u_{i}^{(n-\rho-1)}(0)\right) \succeq 0
$$


Note that both $y(\cdot)$ and $u(\cdot)$ can be read as outputs of a linear time-invariant system of the form $\dot{\xi}(t)=F \xi(t), \eta=H \xi(t)$; moreover the dimension of the underlying state space is at most $n$. So in case (4.14) holds, we get that $u_{i}(\cdot)=0$ and either (if the inequality in (4.14) is strict) $y_{i}(t)>0$ for small $t$, or $y_{i}(\cdot)=0$. In case $(4.15)$ holds, we obtain $y_{i}(\cdot)=0$ so that index $i$ is active. From our assumptions it then follows that the system evolves on a state space of dimension at most $n-\rho$, so that the inequality in (4.15) is enough to conclude that either $u(t)>0$ for small $t$ (in case strict inequality holds), or $u(\cdot)=0$. We see that in all cases the inequality constraints of mode $I$ are satisfied by $(x(\cdot), y(\cdot), u(\cdot))$, so that it is really a smooth continuation in mode $I$. The uniqueness follows directly from the general Theorem 3.4.

A special feature of the linear setting is that it allows a frequency-domain approach to the mode selection problem. To see this, note that to a strictly proper rational vector function $y(s)$ we can associate the coefficients $y^{j}$ of its power series expansion around infinity

$$
y(s)=y^{0} s^{-1}+y^{1} s^{-2}+y^{2} s^{-3}+\cdots
$$

and, as is easily verified, the lexicographic nonnegativity condition $\left(y^{0}, y^{1}, \ldots\right) \succeq 0$ is equivalent to the condition

$$
y(s) \geq 0 \text { for all sufficiently large } s \text {. }
$$

Moreover, when two strictly proper functions $y(s)$ and $u(s)$ are related via

$$
y(s)=C(s I-A)^{-1} x_{0}+\left(D+C(s I-A)^{-1} B\right) u(s)
$$

then, as is again easily verified, the corresponding coefficients $\left(y^{0}, \ldots, y^{n-1}\right)$ and $\left(u^{0}, \ldots, u^{n-1}\right)$ are related in exactly the same way as in the LDCP. We are therefore motivated to consider the following problem, which we shall call the rational complementarity problem.

Problem RCP. Let matrices $A, B, C, D$ of sizes $n \times n, n \times k, k \times n$, and $k \times k$ respectively be given. Define rational matrix functions $T(s)$ of size $k \times n$ and $G(s)$ of size $k \times k$ by $T(s)=C(s I-A)^{-1}$ and $G(s)=C(s I-A)^{-1} B+D$. For given $x_{0}$, find strictly proper rational functions $y(s)$ and $u(s)$ such that the equality

$$
y(s)=T(s) x_{0}+G(s) u(s)
$$

holds, and there exists an $s_{0} \in \mathbb{R}$ such that for all $s \geq s_{0}$ we have

$$
y(s) \geq 0, \quad u(s) \geq 0, \quad y(s)^{T} u(s)=0 .
$$

The following theorem shows that the problems RCP and LDCP are equivalent.

TheOREM 4.2 For given data $A, B, C, D$, and $x_{0}$, the problem LDCP has a solution if and only if the problem $R C P$ has a solution.

Proof Suppose first that RCP has a solution $(y(s), u(s))$; write $y(s)=y^{0} s^{-1}+y^{1} s^{-2}+\cdots$ and $u(s)=u^{0} s^{-1}+u^{1} s^{-2}+\cdots$. From (4.18) it follows that (4.11) holds. Clearly (4.19) implies that for all $i=1, \ldots, k$ and for all $s \geq s_{0}$ either $y_{i}(s)=0$ or $u_{i}(s)=0$ or both. Now $y_{i}(s)$ and $u_{i}(s)$ are rational functions and so they vanish either only at a finite number of points or identically. It follows that we actually have $y_{i}(s) \equiv 0$ or $u_{i}(s) \equiv 0$ or both. If $y_{i}(s)$ or $u_{i}(s)$ is nonzero, then the inequalities in (4.19) guarantee that at least one of (4.12) and (4.13) will be satisfied.

Conversely, suppose that LDCP has a solution. Then, by Thm.4.1, the problem (4.10)-(3.2) has a unique smooth solution $(x(\cdot), y(\cdot), u(\cdot))$ with initial condition $x(0)=x_{0}$. The time functions $y(\cdot)$ and $u(\cdot)$ can both be read as outputs of a finite-dimensional linear system and hence their Laplace transforms, to be denoted (with some abuse of notation) by $y(s)$ and $u(s)$, are rational. Moreover, by 
the fact that $(x(\cdot), y(\cdot), u(\cdot))$ satisfies $(4.10)$ and $(3.2)$ with initial condition $x(0)=x_{0}$ it follows that $y(s)$ and $u(s)$ satisfy the conditions of RCP.

Note that the conditions (4.17) and (4.19) can be looked at as an LCP for any fixed and sufficiently large value of $s$. But of course we do not know in advance which values of $s$ are 'sufficiently large'; indeed, this depends in particular on $x_{0}$. Nevertheless, if some value of $s$ is selected and the corresponding LCP is solvable so that it determines an active index set, this index set may be tested for correctness on the LDCP, which becomes a set of linear equations once the index set has been fixed. If the candidate fails, a larger value of $s$ can be chosen and the test can be repeated.

To illustrate the use of frequency-domain methods, consider once more the example of the Introduction. First note that a complementary-slackness formulation of the dynamics of this example can be written as

$$
\begin{aligned}
& \ddot{x}_{1}(t)=-2 x_{1}(t)+x_{2}(t)+u(t) \\
& \ddot{x}_{2}(t)=x_{1}(t)-x_{2}(t) \\
& y(t)=x_{1}(t) \\
& y(t) \geq 0, \quad u(t) \geq 0, \quad y(t) u(t)=0 .
\end{aligned}
$$

This gives rise to the following equations:

$$
\begin{aligned}
& \left(s^{2}+2\right) x_{1}=x_{2}+u+x_{30}+s x_{10} \\
& \left(s^{2}+1\right) x_{2}=x_{1}+x_{40}+s x_{20}
\end{aligned}
$$

in which the vector $\left(x_{10}, x_{20}, x_{30}, x_{40}\right)$ represents an initial condition. The variable $x_{2}$ can be eliminated by multiplying the first equation by $s^{2}+1$ and then using the second equation. This leads to

$$
\left(s^{2}+1\right)\left(s^{2}+2\right) x_{1}=x_{1}+x_{40}+s x_{20}+\left(s^{2}+1\right)\left(u+x_{30}+s x_{10}\right)
$$

or

$$
\left(s^{4}+3 s^{2}+1\right) x_{1}=\left[s\left(s^{2}+1\right), \quad s, \quad s^{2}+1, \quad 1\right]\left[\begin{array}{c}
x_{10} \\
x_{20} \\
x_{30} \\
x_{40}
\end{array}\right]+\left(s^{2}+1\right) u
$$

For each fixed $s$ there is an associated scalar LCP. Although it is trivial, let us note that the equations

$$
y=x+u, \quad y \geq 0, \quad u \geq 0, \quad y u=0
$$

are uniquely solved as follows: if $x>0$ then $u=0, y=x$; if $x<0$ then $y=0, u=-x$; if $x=0$ then $y=u=0$. Taking this into account, it is seen that the equation (4.21) with the associated inequality constraints leads to the following solution of the mode selection problem. Since at the instant of collision always $x_{10}=0$, the selection problem is dominated firstly by the sign of $x_{30}$. If this sign is positive, then the mode with inactive constraint will be selected, whereas the mode with active constraint will be selected (and will give rise to an impulsive solution) if the sign is negative. However, if $x_{30}=0$, then the highest power of $s$ is associated with $x_{20}$ and so it will be the sign of this quantity that will determine which mode is chosen. Again, if the sign of $x_{20}$ is positive, then the mode with inactive constraint will be selected, and if the sign is negative, then the other mode will be selected. If also $x_{20}=0$ then the sign of $x_{40}$ becomes decisive. Finally if $x_{40}$ vanishes as well then the system is at rest, a situation which belongs to the constrained mode as well as to the unconstrained mode. 


\section{Conclusions}

The interaction of discrete and continuous elements can lead to extremely complex models. One way of beating the potential complexity is by the introduction of what one might call 'formalisms', that is, sets of high-level rules that allow a compact specification of the dynamics of a hybrid system. The trade-off is of course that the use of a formalism reduces flexibility; one cannot expect to model every hybrid system within a given formalism. By the existence of this trade-off, it is to be expected that various formalisms at various levels of generality may co-exist with each other and will be used as alternatives, depending on the concrete situation at hand. The use of formalisms also will help the development of theory since it adds structure to the rather wide notion of a "hybrid system".

In this paper we have discussed a formalism which we have called the complementarity formalism. The formalism appears to be suited for mechanical systems with unilateral constraints, electrical networks with diodes, and the Hamiltonian equations for optimal control problems with inequality constraints, and we have shown here that the formalism applies also to systems with relays and systems with saturation. The fact that the presence of both discrete and continuous aspects creates many new problems is vividly illustrated by the observation that even the well-posedness of the dynamic systems within the complementary formalism needs extensive study. In this paper we have considered only part of this problem, namely the uniqueness of smooth continuations and the associated mode selection problem. We have given several alternative problem formulations in algebraic and functional form, and we presented sufficient conditions for uniqueness of smooth continuations to hold.

It should be clear that a full development of the complementarity formalism will take much more work than has been presented here. The reader may already have noted that some of the examples we have presented in section 2 actually do not fit the setting that was used in section 3 and later. Because of the introduction of new variables, a redundancy in the 'output' variables (constraints) is created that is reflected in a nondeterminacy of the 'input' variables (multipliers); as a consequence, Assumption 3.2 needs to be reformulated. These difficulties are not essential however as will be shown in forthcoming work; it should also be noted that Lötstedt [11] already dealt with dependent constraints in his treatment of mechanical systems with inequality constraints.

The well-posedness issue concerns more than just the uniqueness of smooth continuations; one also has to consider the uniqueness of jumps, and one has to guarantee that only a finite number of jumps can occur within a given instant (no 'chattering' at infinite speed). Furthermore the existence of solutions from each feasible point should be shown. In the classical theory of continuous dynamical systems, the notion of well-posedness usually refers also to continuous dependence on initial conditions; it would be of interest to investigate this also for complementary-slackness systems. Among the further basic dynamic properties that call for investigation, the stability of equilibria is probably the most important one; the fact that the energy concept still plays a major role for mechanical systems with inequality constraints would seem to suggest that a Lyapunov approach might be feasible for systems described by the complementarity formalism. An interesting question is whether stability results so obtained may be of help in the design of hybrid systems.

As already stressed in the paper, well-posedness is not just a theoretical matter, but is closely related to basic issues that are of importance in the simulation of complementary-slackness systems. There is a need to develop algorithms for all the phases mentioned at the beginning of section 3 , in continuation of the work that has already been done in connection with DAEs, the solution of optimal control problems with inequality constraints, the simulation of electrical networks, and multibody dynamics. Work is also needed to compare the setting of discontinuous dynamics with other approaches, in particular the smoothing methods that are often applied in simulation. An element such as a relay or an ideal diode is usually an approximation of a situation that actually has smoother characteristics, and one has to weigh the advantages and disadvantages of non-smooth modeling versus those of smooth modeling. Arguments that may play a role here include the new types of mathematical problems that are brought in by non-smooth modeling and the associated complexity of programming, the difficulties that variable-stepsize algorithms may have with steep parts of element characteristics, the complexity 
of physical modeling, and the desired degree of approximation. A comparison between smooth and non-smooth modeling may also be of interest from a theoretical point of view; for instance the example mentioned briefly in section 3 of an ill-posed system with relay may suggest that there is a connection between ill-posedness and instability of equilibria of a corresponding 'smoothed' system.

In section 2 we have shown that the extent of complementarity modeling includes for instance systems with relays or saturation effects, and it was already suggested that piecewise linear characteristics might more generally be included in the complementarity formalism. It will be of interest to further explore the range of complementarity modeling. For some systems an extension of the framework will be called for, for instance when the switching between the alternatives that are typical for complementarity modeling is not triggered by the variables that are involved in these alternatives, but by other variables that may be related to the continuous state or to an external (discrete) input. An extension along a somewhat different line would be the inclusion of hysteretic effects. It is to be expected that

such extensions will bring complementary-slackness systems and their variants closer to other classes of hybrid systems.

\section{REFERENCES}

[1] K. E. Brenan, S. L. Campbell and L. R. Petzold (1989). Numerical Solution of InitialValue Problems in Differential-Algebraic Equations. North-Holland, Amsterdam.

[2] H. Chen and A. Mandelbaum. Leontief systems, RBV's and RBM's. In Applied Stochastic Analysis, Stochastics Monographs, vol.5, M. H. A. Davis and R. J. Elliott (eds.). Gordon \& Breach, New York, 1991, pp. 1-43.

[3] R. W. Cottle, J.-S. Pang and R. E. Stone (1992). The Linear Complementarity Problem. Academic Press, Boston.

[4] B. C. Eaves And C. E. Lemke (1981). Equivalence of LCP and PLS. Mathematics of Operations Research 6, 475-484.

[5] F. R. Gantmacher (1959). The Theory of Matrices (Vol. I). Chelsea, New York.

[6] C. W. GEAR (1988). Differential-algebraic equation index transformations. SIAM J. Sci. Stat. Comput. 9, 39-47.

[7] P. T. Harker And J.-S. PANG (1990). Finite-dimensional variational inequality and nonlinear complementarity problems: A survey of theory, algorithms and applications. Math. Prog. (Ser. B) $48,161-220$.

[8] T. Kailath (1980). Linear Systems. Prentice-Hall, Englewood Cliffs, N.J.

[9] C. W. Kilmister and J. E. Reeve (1966). Rational Mechanics. Longmans, London.

[10] M. KuiJPer (1994). First-Order Representations of Linear Systems. Birkhäuser, Boston.

[11] P. Lötstedt (1982). Mechanical systems of rigid bodies subject to unilateral constraints. SIAM Journal of Applied Mathematics 42, 281-296.

[12] H. NiJmeiJer AND A. J. VAN DeR Schaft (1990). Nonlinear Dynamical Control Systems. Springer-Verlag, Berlin.

[13] J. PÉRÈs (1953). Mécanique Générale. Masson \& Cie., Paris.

[14] M. SpIVAK (1979). A Comprehensive Introduction to Differential Geometry, Vol.I (2nd ed.). Publish or Perish, Berkeley. 
[15] A. W. Tucker (1963). Principal pivotal transforms of square matrices. SIAM Review 5, 305, (Abstract of a lecture delivered at the 1963 SIAM Spring Meeting, Menlo Park, CA.).

[16] A. J. Van der Schaft and J. M. Schumacher (November 1995). The complementaryslackness class of hybrid systems. Report BS-R9529, CWI, Amsterdam, URL: http://www.cwi.nl/ftp/CWIreports/BS/BS-R9529.ps.Z.

[17] J. C. Willems (1972). Dissipative dynamical systems. Part I: General theory. Arch. Rational Mech. Anal. 45, 321-351. 